Sharif University of Technology
Scientia Iranica
Transactions E: Industrial Engineering
hCIttp://scientiairanica.sharif.edu
IRAN I CA

\title{
Environmental effects in an integrated hub location and pricing problem
}

\author{
M. Mohammadi and H. Karimi* \\ Department of Industrial Engineering, University of Bojnord, Bojnord, P.O. Box 94531-55111, Iran.
}

Received 30 April 2019; received in revised form 30 April 2020; accepted 1 June 2020

\section{KEYWORDS}

Pricing;

Hub location;

Environmental costs;

Logit model;

Genetic algorithm.

\begin{abstract}
The product pricing decision is one of the important factors in the profitability of organizations, which has a key role in their survival. Moreover, pricing is determined based on the demand and location of applicants. Therefore, the location of facilities and services influences the pricing. Also, the location problem is a critical issue in the survival of an organization. Furthermore, the hub location problem is a type of location problems that has many applications and saves time and money. On the other hand, location is impossible without considering transportation, and transportation has many negative effects on the environment, such as greenhouse gas emissions, air pollution, noise, etc. For this reason, it is important to consider the environmental costs to reduce the adverse effects. This study considers integrated pricing and hub location problem with environmental costs in the competitive market where customer choice is calculated according to the Logit Model (LM). We use Genetic Algorithm (GA) to solve and observe the environmental cost, entrant profit, incumbent income, impact of customer sensitivity, and discount between hubs on the entrant profit. As the final point, the computational experiments demonstrate that the suggested GA is both efficient and effective.
\end{abstract}

(C) 2022 Sharif University of Technology. All rights reserved.

\section{Introduction}

Price is a measurement and criterion and pricing is just determining the value of goods and services. Pricing is a common practice with a considerable impact on the profit and loss of an organization. Also, a correct pricing policy is effective in survival, cost coverage, and expanded sales, which in turn translates into a larger market share.

Evidence suggests that determining a place to build an industrial plant is one of the basic decisions of investors that can play an essential role in directing the strategic orientation of the factory, as it affects

\footnotetext{
*. Corresponding author. Tel.: +985842284611;

Fax: +985842410700

E-mail address: h.karimi@ub.ac.ir (H. Karimi)
}

the profitability and survival of the organization. For example, if the initial resources of an organization are bulky, the factory should be in the vicinity of the suppliers of raw materials. Moreover, when products are bulky, the factory should not be far from customers. In addition to the economic impact, the selection principle or non-principle location can affect the cultural, social, and environmental aspects. Location is generally seen as a decision closely related to the demand, which in turn is primarily associated with prices. The factors affecting product pricing are generally divided into two internal and external groups. One of the external factors is market properties with the marketplace and the number of customers serving as two sub-branches of market properties [1]. Therefore, the price of products and services is determined based on the demand and location of customers. In conclusion, location and pricing can be mutually interrelated. Location is a long-term decision and pricing appears to be a short- 
term decision; in fact, it represents a continuous process. For this reason, the organization needs to consider the issues of optimal location and appropriate pricing together.

The location decision aims to find the best site for facilities, factories, or companies to reduce costs. Nonetheless, connecting all nodes could be costly and time-consuming and reduce the efficiency of the model. Hence, it is essential to incorporate a network design in the location problems. To overcome this problem, the hub-and-spoke network topology is used. A key feature of this network is that it can be applied to indirect and fewer connections between Origin/Destination (O/D) pairs using hub nodes. The hub nodes are tasked with the collection, transshipment, and distribution. Also, the connection of two hub nodes is moderated with a discount factor, which is shown by $0 \leq \alpha \leq 1$. Therefore, reducing connections and incorporating the discount factor between hub nodes can reduce overall transportation costs.

Hub Location Problems (HLPs) typically involve locating hub nodes and non-hub nodes as well as allocating non-hub nodes to hub nodes with spoke links. The objective of this problem is to minimize costs and distance or maximize profit. In fact, in HLPs, location and network design decisions are combined. Hub location is important for a wide range of scientific and industrial processes such as transportation [2], postal services [3], and telecommunications [4]. One of the best functional examples of pricing at HLP is the airline industry. Pricing at the airport is considered during the planning horizon. Although a daily route may have different pricing depending on the pricing policy of the airport, its pricing over a planning horizon indicates the average of all prices during that period. Naturally, hub airports have a greater demand than non-hub airports. As a result, the number of flights and the need for new airplanes will be higher, as will be the amount of required fuel. In addition to optimizing flights schedule, the probability of increasing demand for an airport runway should be considered. Therefore, problems such as runway pavement, resource consumption, noise, and Greenhouse Gas (GHGs) emissions induced by extra flights, all of which hurt the ecosystem, should be considered. Among these deleterious effects, GHGs emissions, especially $\mathrm{CO}_{2}$ is the most concerning [5]. Also, in addition to affecting human health and ecosystem, air pollution impacts nations' economic dimensions; these effects on housing prices are studied by Chen and Shiyi [6].

Since routing is an integral part of location decision, and vehicles can damage the environment and human health, it is important to consider environmental costs through the optimal use of vehicles by choosing the optimal path as part of the pricing decision at HLPs. The pollution generated by a vehicle depends on its load and speed, among other factors [7].

There is a paucity of research on the impact of environmental costs on hub location with few studies considering pricing at hub location together with environmental costs. Hence, we consider integrated pricing and HLP with environmental costs in this paper. The competitive market is studied because it resembles the real world and special discriminatory pricing policy. The price-sensitive demand is more compatible with real-life conditions. The process of customer choice is modeled using a Logit Model (LM), which is mostly used for representing a discrete choice. One of the major attributes of this model is matching different features of options. For example, some variables associated with transportation include cost, waiting time, travel time, type of car, and price. This model also has a sensitivity parameter denoted by $\Theta$. A large $\Theta$ implies that consumers are sensitive to price and they concentrate on less expensive paths, and vice versa (for more studies on LMs, see [8,9]). Also, this paper gives an account of environmental costs and analyzes the impact of $\Theta$ and different discount factors $\alpha=[0.2,0.4,0.6,0.8,1]$ on the entrant's profit and the incumbent's (i.e., existing company) income. It is assumed that the incumbent charges its costs plus an additional percentage to their customers (i.e., mill pricing). When customers transport the product from the firm to their warehouses or other places, the pricing strategy is mill pricing. In this case, the full price for all customers irrespective of their locations is equal to mill price, plus transportation cost. However, in the uniform pricing, customers pay the same price regardless of distance; however, ordinarily, the firm may refuse to transport beyond a certain maximal range [10]. We do not consider the incumbent's profit as it is assumed to have existed in the market for a while and therefore, its investment costs have shrunken.

The rest of this paper proceeds as follows: Section 2 presents an overview of the recent studies. Section 3 describes the problem and model formulation. Sections 4 and 5 present the statistical analysis using the Civil Aeronautics Board (CAB) dataset and discussion, respectively. Finally, conclusions are drawn in Section 6.

\section{Literature review}

In this section, a review of relevant literature is provided. Goldman [11] was the first to introduce the HLP network, but the first mathematical formulation of an HLP was presented by O'Kelly [12]. In the classical hub location models, it is assumed that all hub nodes are completely connected via hub links that are discounted by a constant factor. Furthermore, in the classic models, non-hub nodes are not connected directly. A new approach to HLPs called hub arc location 
was introduced by Campbell et al. [13,14]. In this approach, the assumption that all hubs are connected is relaxed, generating some unrealistic results in real-life applications [15].

As mentioned earlier, HLP is used in many areas. Nickel et al. [16] introduced the first mixedinteger programming models for public transportation networks. They suggested two models: Public Transport (PT) and Generalized Public Transport (GPT). Then, Gelareh and Nickel [17] expanded PT into Hub Location Model for Public Transport (HLPPT) by reducing the number of variables and constraints.

Generally, solutions can be categorized into exact and approximation methods. Some of the exact methods include game theory [18], branch and bound [19], and dynamic programming [20]. One of the approximation methods is metaheuristic. Different algorithms have been proposed to be metaheuristic including tabu search algorithm [21], Genetic Algorithm (GA) [22], and variable neighborhood search algorithm [23].

For further studies on hub location with alternate assumptions in the transportation area, see [24,25]. The objective of most studies in the hub location is to minimize costs and travel time or maximize profit and service level. Some researchers such as Campbell [15] have integrated both cost and service. However, the location decision is not the only profitable component of the company and pricing plays a critical role in profitability. One of the most notable examples is pricing at 27 large US airports, which helped solve the delay problem of 13 passenger-years and 1000 aircrafthours and save 3-5 million dollars daily [26].

There are a host of studies on pricing-location and HLPs. In the following, some studies on pricing hub locations are reviewed. Spiller [27] was one of the first to study the pricing of the hub and spoke networks in the monopoly market at airlines. Daniel [28] modeled a congestion pricing problem at large hub airports that accounted for the capacity of hubs and stochastic queues. Oum et al. [29] examined marginal pricing policy over a network of hub-and-spoke airports. Another paper on pricing at hub airports was the study undertaken by Nero and Black [30]. This article differed from another relevant research in considering environmental costs. Vowles [31] explored the determinants of airfare prices in the hub-to-hub market.

Moreover, some studies described in the following have been researched on the environmental aspect of hub location. O'Kelly considered the environmental costs and benefits of using hubs [32]. He worked on fuel burn as an indicator of environmental cost. Loo et al. worked on the impact of the environmental aspects of hub nodes in air transportation [33]. They used data of Greek and Hong Kong/Sanya flight information. Niakan et al. considered a multi-objective model in which two objectives in this model minimize fuel consumption and greenhouse emissions costs [34]. As another research, Zhalechian et al. presented a multiobjective model [35]. An objective of their model minimized pollution costs.

Simultaneous consideration of hub location and pricing problems was first undertaken by Lüer-Villagra and Marianov [22]. In their model, the number of hubs, prices, and locations were determined.

The factors affecting carrier pricing behavior were studied by Zhang et al. [36]. They investigated the effect of hub hierarchy on the airfare pricing in a competitive market. Lin and Zhang [37] analyzed the discriminatory pricing of the airfare on the hub-andspoke network. An analysis of the discriminatory pricing in a hub airport, which accounted for congestion, scheduling, and capacity investment, was conducted by Lin and Zhang [38].

However, hub networks make extra environmental costs, which have been discussed in the literature. What is not yet clear is the impact of environmental costs on pricing in the HLP. Therefore, the main contribution of this paper is modeling an integrated hub location and pricing problem with environmental costs. To increase compatibility with real-world applications, some aspects such as single allocation, special discriminatory pricing, and competitive market were considered and the LM was used for modeling the process of customer choice.

\section{Model formulation}

One of the best examples of application-based pricing in hub location is the ground fright services. These prices are determined by factors such as the number of competitors, the load of frights, and the distance between the origin and destination nodes. As is known, in this service, capacity and demand at hub nodes are higher than those at other nodes. As a result, the number of transportation and the need for new vehicles is increasing at hub nodes. This issue results in more fuel consumption. Perhaps, there is also a need for a new freeway or highway and maintenance of asphalt. Therefore, more fuel and GHGs are added as a result of added transportation. All of these have adverse effects on the ecosystem. In this application, vehicle speed and acceleration are different in each transportation according to the characteristics of the road. Hence, in the following, we consider a hub-and-spoke network in the competitive market in which the first company (incumbent) operated. The second company (entrant) desired to enter this market and maximize its profit by choosing the optimum hub-and-spoke network and prices. The incumbent used a hub network topology. The entrant's price covered costs in addition to a fixed profit percentage. Both entrant and incumbent could use common hub nodes, but this led to congestion. As 
a result, the incumbent and entrant's hub nodes were different from each other. as follows:

Some of the basic assumptions of this model are

- All incumbent and entrant's hubs are interconnected;

- The discount factor is considered for inter-hub connections and non-hub nodes are forbidden;

- The customer decision process is modeled by an LM;

- The environmental costs depended on the speed of the vehicle;

- A single allocation strategy is employed for the entrant;

- The hub nodes of the incumbent are specified;

- Mill pricing strategy is considered for an incumbent;

- A completed hub network is considered.

In the following, first sets, indices, parameters, and variables are defined and then the model is described.

sets:

- N: Set of all nodes;

- P: Set of incumbent hubs.

\section{Indices:}

- $i, j$ : Origin and destination nodes;

- $\quad k, m$ : Entrant hub nodes.

\section{Parameters:}

- $q_{i j}$ : Demand between origin $i$ and destination $j$;

- $F_{k}$ : Fixed cost of opening a hub node;

- $\alpha$ : Discount factor between hub nodes;

- $\chi$ : Discount factor between non-hub origin and hub destination;

- $\partial$ : Discount factor between hub origin and non-hub destination;

- $d_{i j}$ : Distance between $i$ and $j$;

- $c_{f}$ : Unit cost of fuel;

- e: Unit cost of GHGs emitted;

- $a$ : Acceleration of the vehicle $\left(\mathrm{m} / \mathrm{s}^{2}\right)$;

- $g$ : Gravitational constant $\left(9.81 \mathrm{~m} / \mathrm{s}^{2}\right)$;

- $\theta_{i j}$ : Road angle between nodes $i$ and $j$;

- $v_{i j}$ : Speed of the vehicle between nodes $i$ and $j$;

- $c_{r}, c_{d}$ : Coefficients of rolling resistance and aerodynamic drag;

- $\rho$ : Air density $\left(\mathrm{kg} / \mathrm{m}^{3}\right)$;

- $\lambda_{i j}$ : The arc specific constant calculated using Eq. (1) [7]:

$$
\lambda_{i j}=a+g \sin \theta_{i j}+g C_{r} \cos \theta_{i j} \quad \forall i, j \in N .
$$

- $A$ : Frontal surface area of the vehicle $\left(\mathrm{m}^{2}\right)$;

- $\beta$ : A vehicle-specific constant calculated using Eq. (2) [7]:

$$
\beta=0.5 c_{d} A \rho
$$

- $\quad c_{i j}$ : Variable cost per unit of the flow between nodes $i$ and $j$, which is obtained through Eq. (3):

$$
c_{i j}=\left(c_{f}+e\right) \lambda_{i j} d_{i j} \quad \forall i, j \in N
$$

- $c_{i j k m}$ : Variable cost per unit of the flow between nodes $i$ and $j$ that passes through one or two hub(s) and is achieved using Eq. (4):

$$
\begin{aligned}
& c c_{i j k m}=\chi \cdot c_{i k}+\alpha \cdot c_{k m}+\partial \cdot c_{m j} \\
& \quad \forall i, j, k, m \in N .
\end{aligned}
$$

- $k_{i j}$ : The fixed cost per unit of the flow for each arc between nodes $i$ and $j$;

- $\Theta$ : The sensitivity parameter of the LM;

- delta $_{i j s n}(i, j \in N, s, n \in P)$ : The extra percentage charged by the incumbent between origin and destination nodes makes profits. This percentage may be different for each path and it is assumed that these values are produced uniformly at $[0.1,0.4]$, which is inspired from [22];

- $\bar{p}_{i j s n}$ : Incumbent price, which is obtained from Eq. (5). This equation is defined according to the mill pricing strategy which is defined as an assumption:

$$
\begin{gathered}
\bar{p}_{i j s n}=\left(1+\operatorname{delta}_{i j s n}\right) \cdot c c_{i j s n}, \\
\forall i, j \in N, s, n \in P .
\end{gathered}
$$

- $\eta_{i j}$ : This parameter is obtained from Eq. (6):

$$
\eta_{i j}=\sum_{s, n \in P} \exp \left(-\Theta \bar{p}_{i j s n}\right) \quad \forall i, j \in N
$$

\section{Variables:}

- $x_{i j k m}$ : The fraction of flow between $i$ and $j$ served via entrant's hub nodes $k$ and $m .(i, j, k, m \in N)$;

- $p_{i j k m}$ : The entrant's price to transfer one unit of flow between $i$ and $j$ through hub nodes $k$ and $m$. $(i, j, k, m \in N)$;

- $h_{i k}=1$ : If the entrant makes a connection between the non-hub node $i$ and hub node $k ; 0$ : otherwise. $(i, k \in N)$;

- $y_{k}=1$ : If the entrant creates one hub at node $k ; 0$ : otherwise. $(k \in N)$. 


\section{Description of the model:}

$$
\begin{aligned}
& \max \sum_{i, j, k, m \in N}\left(p_{i j k m}-c_{i j k m}\right) q_{i j} x_{i j k m} \\
& -\sum_{i, k \in N} k_{i k} h_{i k}-\sum_{k \in N} F_{k} y_{k} \\
& -\left[\sum_{i, k \in N}\left(c_{f}+e\right) d_{i k} \beta\left(v_{i k}\right)^{2} h_{i k}\right. \\
& \left.+\sum_{i, j \in N}\left(c_{f}+e\right) d_{i j} \beta\left(v_{i j}\right)^{2} y_{i} y_{j}\right] \text {, } \\
& x_{i j k m}=\frac{\exp \left(-\Theta p_{i j k m}\right)}{\sum_{s, n \in N}\left(h_{i s} h_{n j} \exp \left(-\Theta p_{i j s n}\right)\right)+\eta_{i j}} \\
& \forall i, j, k, m \in N \text {, } \\
& x_{i j k m} \leq h_{i k} \quad \forall i, j, k, m \in N, \quad i \neq j, \\
& x_{i j k m} \leq h_{j m} \quad \forall i, j, k, m \in N, \quad i \neq j, \\
& \sum_{k \in N} h_{i k}=1 \quad \forall i \in N, \\
& h_{i k} \leq y_{k} \quad \forall i, k \in N \\
& h_{i k} \leq 1-y_{i} \quad \forall i, k \in N, \quad i \neq k, \\
& y_{k} \in\{0,1\} \quad \forall k \in N \text {, } \\
& h_{i k} \in\{0,1\} \quad \forall i, k \in N \text {, } \\
& p_{i j k m} \geq 0 \quad \forall i, j, k, m \in N, \\
& x_{i j k m} \geq 0 \quad \forall i, j, k, m \in N .
\end{aligned}
$$

The objective function (Eq. (7)) maximizes the entrant's profit, which comprises the income with fixed and variable costs subtracted. Generally, there are four types of cost including variable costs per unit of flow, fixed costs per unit of flow for each arc, fixed costs of opening a hub node, and environmental costs incurred by the vehicle speed between entrant's hubs. The last term of cost function is inspired by [7]. Constraint (8) shows a fraction of flow or market share served by entrant according to the LM. This market share is earned by prices that entrant wants to consider. Constraints (9) and (10) ensure that origin $i$ and destination $j$ are assigned to the hub nodes $k$ and $m$, respectively, and $x_{i j k m}$ can take a value. Constraints (11) represent a single allocation strategy. Constraints (12) ensure that node $i$ remains connected to node $k$ when $k$ is a hub. Constraints (13) indicate that node $i$ connects to node $k$ when $i$ is nonhub. Finally, the last four constraints exhibit types of decision variables.

As can be seen, this model is mixed-integer nonlinear programming and incorporation of pricing and HLP adds to the complexity of the model. An exact process for a problem of this size would be excessively time-consuming, as four indices must be formulated for the pricing of each path. Therefore, GA is used to resolve this problem. The reasons for choosing a GA are:

1. No need for local search processes, as genetic operators assist the algorithm to discover the solution space;

2. GAs have also had many successes in solving various problems including hub location-based problems (see $[39,40])$;

3. GAs are proven to be having a good and nearoptimal solution [41].

At each step of GA, possible solutions and arc connections are discovered for HLP. Moreover, pricing problems and environmental costs are resolved, and the optimum flows and prices are obtained. Finally, the objective value is calculated after subtracting costs from income. The pricing problem is proved according to [22].

\subsection{Genetic Algorithm ( $G A$ )}

GA is the most popular metaheuristic search technique that is widely used for finding near-optimal (and sometimes optimal) solutions to optimization problems. First, random feasible solutions with population size $\left(n_{\text {pop }}\right)$ in the valid hub-and-spoke networks are created and saved in a solution set $S$. This value in our algorithm is set at 10 using trial-and-error method. Each member of the population is called chromosome and each chromosome consists of several genes. Then, the main loop of the algorithm is initiated. On each iteration of the algorithm, two solutions (called parents) are randomly selected from $S$, and two solutions are generated by applying a single-point crossover operator called offspring. Then, an offspring is mutated with probability $p_{m}$. The objective function is calculated for all produced solutions. In the next step, the previous population produces offspring of crossover, and mutation is merged and sorted according to the objective function so that the first $n_{\text {pop }}$ is chosen. One round of the loop ends in this part and the algorithm keeps iterating until an ending condition has occurred. The components of the GA are described in the following.

\subsubsection{Solution representation}

The solution representation is an important component 


\begin{tabular}{l|l|l|l|l|l|l|l|l|l|l|l|l|l|l|l|l|l|l|l|l|l|l|l|l|}
\hline 1 & 0 & 1 & 1 \\
18 & (a) \\
\hline 18 & 4 & 14 & 4 & 18 & 18 & 14 & 4 & 4 & 14 & 4 & 4 & 18 & 14 & 18 & 4 & 4 & 18 & 14 & 18 & 4 & 14 & 4 & 18 & 14 \\
\hline
\end{tabular}

(b)

Figure 1. Example of the solution representation.

of the GA, as it affects the performance and runtime of the algorithm. Here, we use a binary display and the hub nodes and nodes connections are shown as a binary string. For example, if an entrant's potential hubs set is $\{4,11,14,18\}$ and nodes $\{4,14,18\}$ are chosen as the hub, the solution representation for hub nodes and nodes assignment would be as follows. As shown in Figure 1(a), the value of node cells $\{4,14,18\}$ is equal to one. Also, in the node assignment representation shown in Figure 1(b), the value of the first cell is 18 , meaning that the first node is assigned to the hub node 18 or the fourth hub node.

\subsubsection{Crossover operator}

The basic operator for producing new solutions in GA is a crossover. The crossover operator discovers the solutions for a search space. In this paper, we used the roulette wheel to select parents and single-point crossover. This study used vertical or horizontal cuts in the vector or matrix randomly and produced two new offspring that shared some parts of both parents. If $n h$ is the number of the hubs, a 1-point crossover operator uses a random number $t \in[1, n h-1]$ to cut the parent vectors. If nodes $\{4,11,14,18\}$ are entrant potential hubs, as shown in Figure 2, two parents are selected and a random number of offspring are generated as follows. Also, a random number is generated again for infeasible offspring.

In the first parent, nodes $\{4,14,18\}$ are selected as the hub, meaning that variables $y_{1}, y_{3}$, and $y_{4}$ are equal to 1 . In the second parent, nodes $\{11,18\}$ are selected as the hub. Then, $t=2$ is chosen for random cutting of the point and the offspring is produced. After the cutting of parents, the first offspring would be composed of the first part of the first parent and the second part of the second parent, meaning that nodes $\{4,18\}$ become a hub and variables $y_{1}$ and $y_{4}$ will be equal to 1 . The crossover rate in the suggested GA is set at 0.6 .

\subsubsection{Mutation operator}

The mutation operator makes some changes in genes of the chromosome with low probability. This is intended

\begin{tabular}{|c|c|c|c|c|c|c|c|c|c|}
\hline $\begin{array}{c}\text { First } \\
\text { parent }\end{array}$ & 1 & 0 & 1 & 1 & $\begin{array}{c}\text { First } \\
\text { offspring }\end{array}$ & 1 & 0 & 0 & 1 \\
\hline $\begin{array}{l}\text { Second } \\
\text { parent }\end{array}$ & 0 & 1 & 0 & 1 & $\begin{array}{l}\text { Second } \\
\text { offspring }\end{array}$ & 0 & 1 & 1 & 1 \\
\hline
\end{tabular}

Figure 2. Example of the single-point crossover.

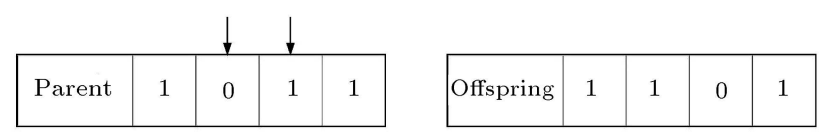

Figure 3. Example of the swap method.

to escape from the local optimum and prevent early convergence. Also, the mutation maintains diversity in the population. In this step, one solution is selected randomly and some chromosome genes are changed. We used the swap method to create a neighborhood. In this method, two random numbers are produced and their genes are moved. An example of a swap method is shown in Figure 3.

As shown in Figure 3, by considering nodes $\{4,11,14,18\}$ as the potential hub set, nodes $\{4,14,18\}$ are chosen as the hub. Then, numbers 2 and 3 are created randomly and the offspring is produced by moving the second and third cells. It should be noted that the mutation rate is set at 0.4 for this GA.

\section{Computational experiments}

For model evaluation, the CAB dataset was used for 25 nodes [42] and $k_{i j}$ was calculated based on the following expression [43]:

$$
k_{i j}=10 \frac{c_{i j} / q_{i j}}{\max _{(k, m) \in P H} c_{k m} / q_{k m}} \quad \forall i, j \in N .
$$

In the parameters related to environmental costs, $c_{d}=$ 0.7 and $A=5$ [44]; $c_{r}=0.012$ [45] and $c_{f}=7 ; e=5$, $g=9.81, p=1.2041$, and $a=0.1$. Also, $\theta_{i j}$ and $\lambda_{i j}$ were obtained from [7]. delta $a_{i j s n}$ was produced uniformly at $[0.1,0.4]$ and the speed of the vehicle was uniform at $[40,100] \mathrm{km} / \mathrm{h}$. Also, $\alpha=\{0.2,0.4,0.6,0.8,1\}$, $\partial=\chi=1$, and $\Theta=\{3.85,5.78,7.7,9.63,11.55,15.39\}$ were also used [22]. It should be noted that for a dataset with 25 nodes to balance the parameters, $k_{i j}$ and $F_{k}$ are divided by 100 . Also, the whole environmental costs are divided by 10000 .

We solved this problem with Basic Open-source Nonlinear Mixed Integer programming (BONMIN) solver and GA programmed in MATLAB 2016 for the first 5 nodes of the dataset using a PC with a Core i5 processor of $2.5 \mathrm{GHz}$ and $4 \mathrm{~GB}$ of RAM with $\alpha=$ $\{0.2,0.4,0.6,0.8\}$ and $\Theta=\{3.85,5.78,7.7,9.63,11.55\}$. The results are shown in Table 1 . In this example, the incumbent's hubs are 2 and 4 . Moreover, the potential entrant's hubs are all five nodes. 
Table 1. Comparison of the results of BONMIN and GA.

\begin{tabular}{|c|c|c|c|c|c|}
\hline \multirow[b]{2}{*}{$\Theta$} & \multirow[b]{2}{*}{$\alpha$} & \multicolumn{2}{|c|}{ GA } & \multicolumn{2}{|c|}{ BONMIN } \\
\hline & & $\begin{array}{c}\text { Solution time } \\
(\mathrm{s})\end{array}$ & Objective & $\begin{array}{c}\text { Solution time } \\
(\mathrm{s})\end{array}$ & Objective \\
\hline \multirow{4}{*}{3.85} & 0.2 & 20 & 8914.2358 & 1306.593 & 8914.2358 \\
\hline & 0.4 & 20 & 8338.9 & 2720.25 & 8338.9 \\
\hline & 0.6 & 24 & 8158.6212 & 2785.63 & 8158.6212 \\
\hline & 0.8 & 69 & 8109.0726 & 2740.54 & 8109.0726 \\
\hline \multirow{4}{*}{5.78} & 0.2 & 61 & 8015.2817 & 2707.462 & 8015.2817 \\
\hline & 0.4 & 53 & 7156.778 & 2683.547 & 7156.778 \\
\hline & 0.6 & 54 & 6363.8319 & 2677.236 & 6363.8319 \\
\hline & 0.8 & 53 & 6172.3868 & 2700.543 & 6172.3868 \\
\hline \multirow{4}{*}{7.7} & 0.2 & 54 & 7482.9668 & 2823.76 & 7482.9668 \\
\hline & 0.4 & 51 & 6478.8546 & 2834.25 & 6478.8546 \\
\hline & 0.6 & 61 & 5800.3765 & 2807.807 & 5800.3765 \\
\hline & 0.8 & 55 & 5470.3055 & 2845.76 & 5470.3055 \\
\hline \multirow{4}{*}{9.63} & 0.2 & 55 & 7302.4297 & 2878.65 & 7302.4297 \\
\hline & 0.4 & 61 & 6197.7196 & 2850.635 & 6197.7196 \\
\hline & 0.6 & 53 & 5982.394 & 2892.483 & 5982.394 \\
\hline & 0.8 & 53 & 5464.2763 & 2897.32 & 5464.2763 \\
\hline \multirow{4}{*}{11.55} & 0.2 & 66 & 7059.131 & 3305.67 & 7059.131 \\
\hline & 0.4 & 56 & 5944.0264 & 3313.78 & 5944.0264 \\
\hline & 0.6 & 59 & 6425.2105 & 3620.673 & 6425.2105 \\
\hline & 0.8 & 59 & 5482.6326 & 3635.318 & 5482.6326 \\
\hline
\end{tabular}

As shown in Table 1, the objective function values obtained from BONMIN and GA are the same, but the solution time of BONMIN and GA is significantly different. The solution time increases by enlarging the size of the problem, and a PC with listed configurations would be unable to solve this problem. As a result, the use of GA would yield better results.

For the analysis of data, the impact of $\alpha, \Theta$ and the ratio of entrant number of the potential hub $(n h)$ to the incumbent hub number (nhmt) on the Entrant Profit (EP) was examined for the CAB dataset using only GA with 10 replications. The average Incumbent
Income (II) (not the profit) was considered because the incumbent was in the market for a while [22]. It is worth mentioning that when $(n h<n h m t)$, nh is considered as the entrant potential hubs; when $(n h>n h m t), n h$ acts as entrant hubs (not potential hubs) because the number of the hub(s) selected by the entrant may be less than nhmt in GA, which contradicts $(n h>n h m t)$. In this research, when $(n h>n h m t)$, nh was $\{4,11,14,18\}$ and $n h m t$ was $\{7,13,17\}$; when $(n h<n h m t), n h$ was $\{11,14,18\}$ and $n h m t$ was $\{4,7,13,17\}$. In that mode, three nodes were selected as the hub for $(\alpha=0.2, \Theta=3.85)$ and 
$(\alpha=0.6, \Theta=3.85)$, and the eighteenth node was selected as the hub in other values of $\alpha$ and $\Theta$.

The coordinate points of applicants, $n h$ and $n h m t$, in the cases of $(n h>n h m t)$ and $(n h<n h m t)$ are shown in Figures 4 and 5, respectively. The computational results included the entrant's profit, the incumbent's income, and environmental costs induced by speed, which are shown in Tables 2 and 3, respectively.

In Tables 2 and 3 , the first column shows the discount factor and the second column presents the sensitivity parameter of the LM. Moreover, the third column shows the Best-Known Solutions (BKS), obtained from the best case of GA. The fourth column shows the average solution of GA. More data such as EP, which is the objective function, Incumbent Income (II), and Environmental Costs Caused by Speed (ECCS) are presented due to depiction of their behaviors according to the average. Column Dev (\%) shows the deviation between the BKS and other solutions. In particular, the column under the heading AVG reports the deviation between the BKS and the average

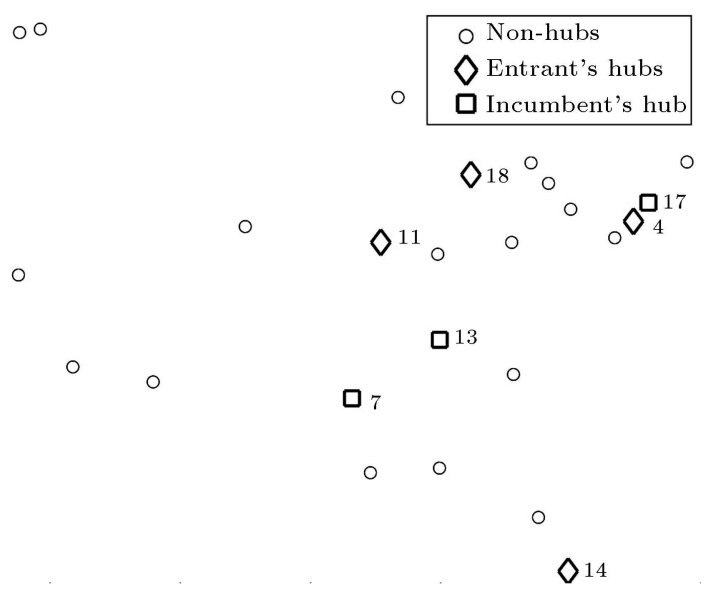

Figure 4. The coordinates of the entrant's nodes and the incumbent's nodes in $(n h>n h m t)$.

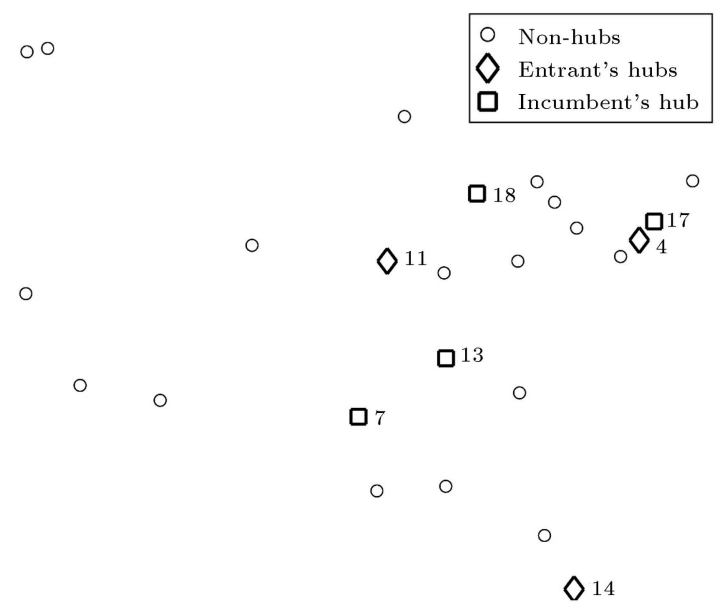

Figure 5. The coordinates of the entrant's nodes and the incumbent's nodes in $(n h<n h m t)$. solution determined by GA. Also, the column WST presents the deviation between the BKS and the GA worst-case solution. It should be noted that in Table 3 , the Dev in more cases is zero due to the use of only one hub in the network. Therefore, finding a solution is easier than other cases. It seems that the GA has obtained an optimal solution in these cases.

As shown in Tables 2 and 3 , in $(n h>n h m t)$, the entrant's profit declines by increasing the values of $\alpha$ and $\Theta$; however, in $(n h<n h m t)$, the entrant's profit for all values of $\alpha$ and $\Theta$ is -1.1698 except in two cases. As a result, in a problem with this assumption, the number of hubs used by the entrant must exceed that of the incumbent hubs. Also, in $(n h>n h m t)$, the entrant's profit is only greater than the incumbent's income in $\Theta=\{3.85,5.78\}$ for all values of $\alpha$. In other words, the entrant can make more profits than the incumbent when customer sensitivity to price is low. A description of the results is given in Figure 6 .

As shown in all charts of Figure 6, with a change of the objective function to $\Theta$ in $(n h>n h m t)$, the entrant's profit declines upon increasing the customer sensitivity to the price for all discount values between hubs.

Figure 7 demonstrates entrant profit decreased by increasing $\alpha$. According to Figure 8(a), the entrant's profit takes a declining process and the incumbent's income takes an incremental process when $\alpha$ increases $(n h>n h m t)$ and $\Theta=7.7$. It should be noted that in $\Theta=7.7$, the entrant's profit is always lower than that of the incumbent, and as mentioned, the entrant's profit in $\Theta=\{3.85,5.78\}$ is higher than the incumbent's income. It should be noted that the entrant's profit is shown in the axis on the left and the incumbent income in the axis on the right. Also, according to Figure $8(\mathrm{~b})$, the entrant's profit remains unchanged $(-1.1698)$ by increasing $\alpha$ in $(n h<n h m t)$ and $\Theta=7.7$, and the incumbent's income takes an incremental trend. In general, the entrant's profit is less than the incumbent income, as shown in Figure 8(a).

A comparison of the incumbent's income in the two modes of $(n h>n h m t)$ and $(n h<n h m t)$ is shown in Figure 9. As can be seen, the incumbent's income in $(n h<n h m t)$ is more than the incumbent's income in $(n h>n h m t)$. It is obvious because the incumbent's ability to serve is greater than that of the entrant in $(n h<n h m t)$. As a result, the incumbent gains a larger market share and generates more revenues.

Figure 10 shows that the environmental costs incurred by speed are significantly lower in the mode of $(n h>n h m t)$ than the mode $(n h<n h m t)$ for different $\alpha$ and $\Theta$. As shown in this figure, the changes in $\Theta$ and $\alpha$ do not result in descending or ascending changes in environmental costs. As expected, in $(n h>n h m t)$, the number of links between non-hubs to hubs is less than $(n h<n h m t)$. Instead of these links, the network 
Table 2. Computational results in $(n h>n h m t)$.

\begin{tabular}{|c|c|c|c|c|c|c|c|}
\hline \multirow[b]{2}{*}{$\alpha$} & \multirow[b]{2}{*}{$\Theta$} & \multirow[b]{2}{*}{ BKS } & \multicolumn{3}{|c|}{ Average } & \multicolumn{2}{|c|}{$\operatorname{Dev}(\%)$} \\
\hline & & & EP & II & ECCS & AVG & WST \\
\hline \multirow{6}{*}{0.2} & 3.85 & 2.2215 & 2.2125 & 0.8335 & 0.5171 & 0.4051 & 1.0232 \\
\hline & 5.78 & 1.1940 & 1.1890 & 0.8329 & 0.5243 & 0.4188 & 1.2870 \\
\hline & 7.7 & 0.6752 & 0.6684 & 0.8345 & 0.4832 & 1.0086 & 1.8518 \\
\hline & 9.63 & 0.3217 & 0.3215 & 0.8352 & 0.5508 & 0.0560 & 0.4791 \\
\hline & 11.55 & 0.1381 & 0.1366 & 0.8344 & 0.5463 & 1.1007 & 1.8838 \\
\hline & 15.36 & -0.1264 & -0.1273 & 0.8319 & 0.5122 & 0.7041 & 1.6124 \\
\hline \multirow{6}{*}{0.4} & 3.85 & 2.2302 & 2.2081 & 0.8829 & 0.5591 & 0.9909 & 1.5312 \\
\hline & 5.78 & 1.1601 & 1.1568 & 0.8845 & 0.4769 & 0.2845 & 0.4541 \\
\hline & 7.7 & 0.6606 & 0.6527 & 0.886 & 0.5526 & 1.2035 & 1.4274 \\
\hline & 9.63 & 0.3276 & 0.3273 & 0.8844 & 0.4447 & 0.1068 & 1.0216 \\
\hline & 11.55 & 0.1071 & 0.1065 & 0.8845 & 0.4775 & 0.5509 & 0.8566 \\
\hline & 15.36 & -0.1515 & -0.1519 & 0.8865 & 0.5398 & 0.2442 & 1.3766 \\
\hline \multirow{6}{*}{0.6} & 3.85 & 2.2065 & 2.1935 & 0.9355 & 0.5591 & 0.5892 & 1.1655 \\
\hline & 5.78 & 1.1802 & 1.1704 & 0.9369 & 0.4769 & 0.8304 & 1.2014 \\
\hline & 7.7 & 0.6431 & 0.6431 & 0.9337 & 0.5526 & 0.0031 & 0.2146 \\
\hline & 9.63 & 0.3454 & 0.3428 & 0.9355 & 0.4447 & 0.7672 & 1.0013 \\
\hline & 11.55 & 0.1391 & 0.1377 & 0.9333 & 0.4775 & 0.9849 & 1.3014 \\
\hline & 15.36 & 0.1231 & 0.1216 & 0.9366 & 0.5398 & 1.2023 & 1.6102 \\
\hline \multirow{6}{*}{0.8} & 3.85 & 2.1153 & 2.1011 & 0.9877 & 0.5635 & 0.6713 & 0.9172 \\
\hline & 5.78 & 1.1826 & 1.1783 & 0.9904 & 0.5577 & 0.3636 & 0.5274 \\
\hline & 7.7 & 0.6434 & 0.6388 & 0.9904 & 0.5337 & 0.7196 & 1.0079 \\
\hline & 9.63 & 0.3057 & 0.3036 & 0.9851 & 0.4808 & 0.6771 & 0.8104 \\
\hline & 11.55 & 0.0858 & 0.0853 & 0.9887 & 0.4602 & 0.6294 & 1.0591 \\
\hline & 15.36 & -0.1010 & -0.1017 & 0.9865 & 0.5035 & 0.6731 & 0.9124 \\
\hline \multirow{6}{*}{1} & 3.85 & 2.2076 & 2.1817 & 1.0376 & 0.4746 & 1.1732 & 1.5241 \\
\hline & 5.78 & 1.1279 & 1.1252 & 1.0376 & 0.6007 & 0.2394 & 0.6474 \\
\hline & 7.7 & 0.6378 & 0.6374 & 1.0366 & 0.4663 & 0.0659 & 0.2063 \\
\hline & 9.63 & 0.33 & 0.3264 & 1.0389 & 0.4921 & 1.1061 & 1.4248 \\
\hline & 11.55 & 0.1006 & 0.0998 & 1.0367 & 0.4353 & 0.7753 & 1.2113 \\
\hline & 15.36 & -0.1284 & -0.1298 & 1.035 & 0.5874 & 1.0746 & 1.8124 \\
\hline
\end{tabular}

Note: BKS: Best-Known Solution; EP: Entrant Profit; II: Incumbent Income; ECCS: Environmental Costs Caused by Speed; AVG: Average solutions that found; WST: Worst-case solution that found.

used hub links. Therefore, in the obtained results for $(n h>n h m t)$, the model causes fewer environmental costs. Furthermore, the trends in $(n h>n h m t)$ and $(n h<n h m t)$ may be the same for each $\alpha$ except in two cases (i.e., $\alpha=0.2, \Theta=5.78$ and $\alpha=0.6, \Theta=5.78$ ). To show the solution convergence in the proposed GA, an instance for $(n h>n h m t)$ with 25 nodes, $\alpha=0.2$ and $\Theta=3.85$ is solved. Figure 11 describes this convergence plot, and Figure 12 shows its solution.

\section{Discussion}

The design of hub networks with the price decision variable is an issue that has been researched rarely in the literature. In this research, a model that integrates pricing and HLP with environmental costs in the competitive market is presented. Computational experiments demonstrate the efficiency of our model in small-sized instances. For large-sized instances, GA is proposed to find an optimal or near-optimal solution with a reasonable computational effort. Tables 2 and 3 show the GA performance. Moreover, the proposed GA explores the hub network and finds feasible solutions. Once a valid hub configuration is found, the pricing problem is solved for this network and the optimal flows and prices are found. Therefore, the performance of GA is dependent on the obtained hub network. Moreover, a sensitivity analysis was conducted to gain a preliminary understanding of how the entrant's or incumbent's profit or income is affected by some changes in parameters such as the number of hubs.

The results show when the entrant's hubs out- 
Table 3. Computational results in $(n h<n h m t)$.

\begin{tabular}{|c|c|c|c|c|c|c|c|}
\hline \multirow{2}{*}{$\alpha$} & \multirow{2}{*}{$\Theta$} & \multirow{2}{*}{ BKS } & \multicolumn{3}{|c|}{ Average } & \multicolumn{2}{|c|}{$\operatorname{Dev}(\%)$} \\
\hline & & & EP & II & ECCS & AVG & WST \\
\hline \multirow{6}{*}{0.2} & 3.85 & -0.0754 & -0.0766 & 1.4426 & 0.6903 & 1.5968 & 2.2541 \\
\hline & 5.78 & -1.1698 & -1.1698 & 1.4411 & 0.8784 & 0.0000 & 0.0000 \\
\hline & 7.7 & -1.1698 & -1.1698 & 1.4391 & 0.8784 & 0.0000 & 0.0000 \\
\hline & 9.63 & -1.1698 & -1.1698 & 1.4412 & 0.8784 & 0.0000 & 0.0000 \\
\hline & 11.55 & -1.1698 & -1.1698 & 1.4450 & 0.8784 & 0.0000 & 0.0000 \\
\hline & 15.36 & -1.1698 & -1.1698 & 1.4462 & 0.8784 & 0.0000 & 0.0000 \\
\hline \multirow{6}{*}{0.4} & 3.85 & -1.1698 & -1.1698 & 1.5336 & 0.8784 & 0.0000 & 0.0000 \\
\hline & 5.78 & -1.1698 & -1.1698 & 1.5310 & 0.8784 & 0.0000 & 0.0000 \\
\hline & 7.7 & -1.1698 & -1.1698 & 1.5306 & 0.8784 & 0.0000 & 0.0000 \\
\hline & 9.63 & -1.1698 & -1.1698 & 1.5373 & 0.8784 & 0.0000 & 0.0000 \\
\hline & 11.55 & -1.1698 & -1.1698 & 1.5301 & 0.8784 & 0.0000 & 0.0000 \\
\hline & 15.36 & -1.1698 & -1.1698 & 1.5306 & 0.8784 & 0.0000 & 0.0000 \\
\hline \multirow{6}{*}{0.6} & 3.85 & -0.7498 & -0.7687 & 1.6185 & 0.6903 & 2.5206 & 3.5142 \\
\hline & 5.78 & -1.1698 & -1.1698 & 1.6285 & 0.8784 & 0.0000 & 0.0000 \\
\hline & 7.7 & -1.1698 & -1.1698 & 1.6216 & 0.8784 & 0.0000 & 0.0000 \\
\hline & 9.63 & -1.1698 & -1.1698 & 1.6198 & 0.8784 & 0.0000 & 0.0000 \\
\hline & 11.55 & -1.1698 & -1.1698 & 1.6195 & 0.8784 & 0.0000 & 0.0000 \\
\hline & 15.36 & -1.1698 & -1.1698 & 1.6188 & 0.8784 & 0.0000 & 0.0000 \\
\hline \multirow{6}{*}{0.8} & 3.85 & -1.1698 & -1.1698 & 1.7113 & 0.8784 & 0.0000 & 0.0000 \\
\hline & 5.78 & -1.1698 & -1.1698 & 1.7078 & 0.8784 & 0.0000 & 0.0000 \\
\hline & 7.7 & -1.1698 & -1.1698 & 1.7123 & 0.8784 & 0.0000 & 0.0000 \\
\hline & 9.63 & -1.1698 & -1.1698 & 1.7110 & 0.8784 & 0.0000 & 0.0000 \\
\hline & 11.55 & -1.1698 & -1.1698 & 1.7065 & 0.8784 & 0.0000 & 0.0000 \\
\hline & 15.36 & -1.1698 & -1.1698 & 1.7109 & 0.8784 & 0.0000 & 0.0000 \\
\hline \multirow{6}{*}{1} & 3.85 & -1.1698 & -1.1698 & 1.7970 & 0.8784 & 0.0000 & 0.0000 \\
\hline & 5.78 & -1.1698 & -1.1698 & 1.7964 & 0.8784 & 0.0000 & 0.0000 \\
\hline & 7.7 & -1.1698 & -1.1698 & 1.7945 & 0.8784 & 0.0000 & 0.0000 \\
\hline & 9.63 & -1.1698 & -1.1698 & 1.794 & 0.8784 & 0.0000 & 0.0000 \\
\hline & 11.55 & -1.1698 & -1.1698 & 1.7976 & 0.8784 & 0.0000 & 0.0000 \\
\hline & 15.36 & -1.1698 & -1.1698 & 1.7950 & 0.8784 & 0.0000 & 0.0000 \\
\hline
\end{tabular}

Note: BKS: Best-Known Solution; EP: Entrant Profit; II: Incumbent Income; ECCS: Environmental Costs Caused by Speed; AVG: Average solutions that found; WST: Worst-case solution that found.

number incumbent's hubs $(n h>n h m t)$, the entrant's profit drops with an increase in $\Theta$ for all values of $\alpha$. In both $(n h>n h m t)$ and $(n h<n h m t)$ modes for an exact $\Theta$, the incumbent's income rises by increasing $\alpha$ and in the second mode $(n h<n h m t)$, the incumbent earns much more income than that in the first mode because of using more hubs and decreasing the transportation costs. However, the entrant's profit is fixed in the second state and this is because the algorithm only selects the node $\{18\}$ from the potential hub nodes $\{11,14,18\}$ for all values of $\alpha$ and $\Theta$; as a result, the entrant's profit does not change. The reason why the algorithm selects just the node $\{18\}$ as a hub can be the lack of capacity constraint for hubs or hub arcs. In other words, if lower bound and upper bound were considered for the capacity of each hub 


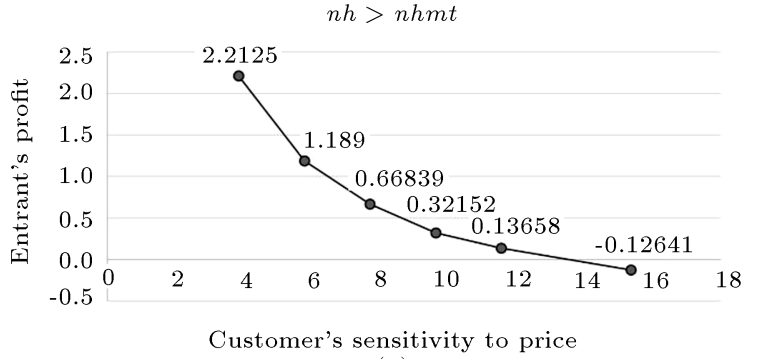

(a)

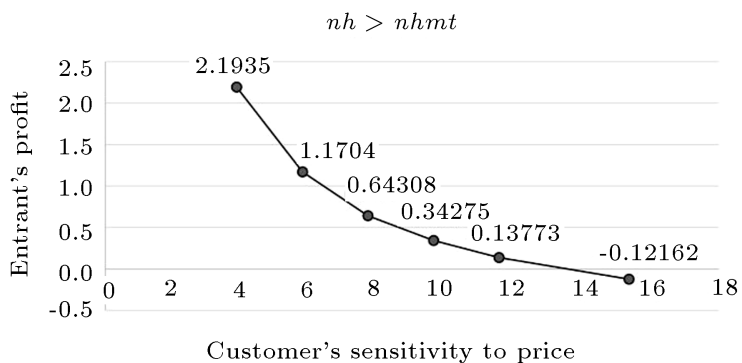

(c)

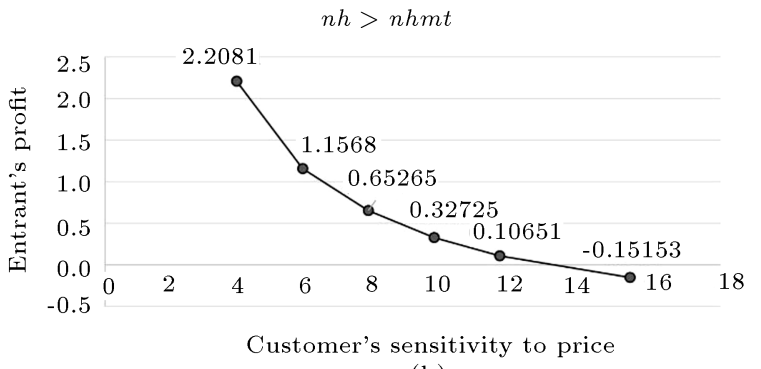

(b)

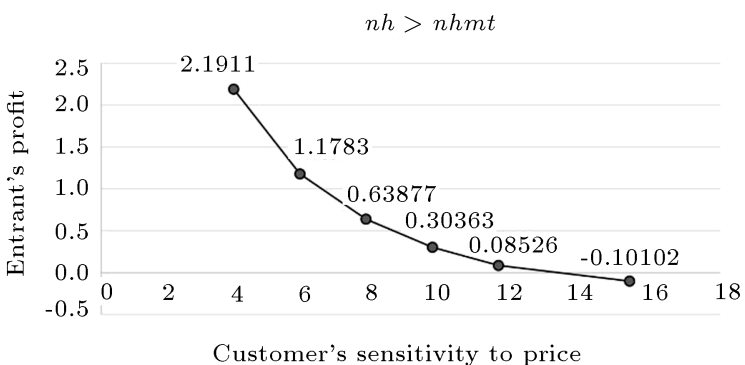

(d)

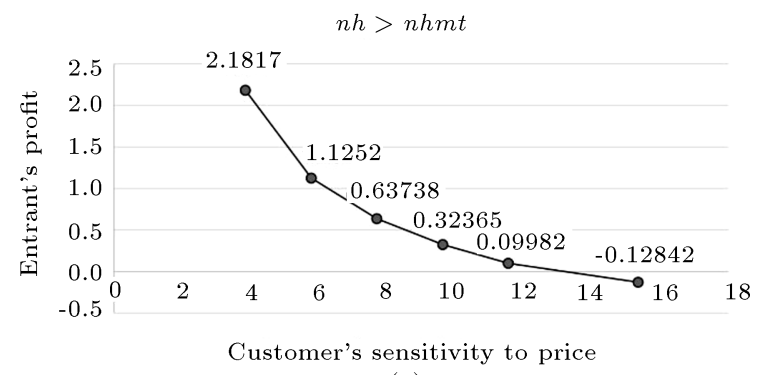

(e)

Figure 6. The objective function changes with variation in the customer's sensitivity to the price at different $\alpha$.

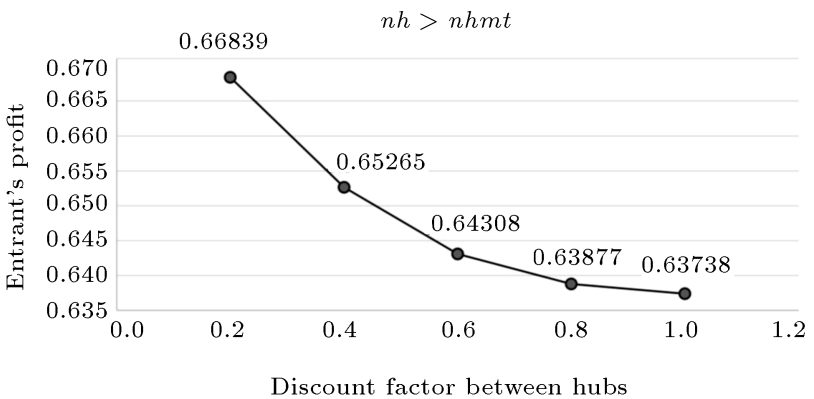

Figure 7. The objective function changes with variation of the discount factor at $\Theta=7.7$.

node or an upper bound was considered for all arcs, the only node $\{18\}$ was not selected as a hub. Also, due to the failure to consider the coverage limit, it is possible that one non-hub node be assigned to the hub farther away through two hubs. For example, for $\alpha=0.2$ and $\Theta=3.85$, the distance between the nonhub node $\{22\}$ and the hub node $\{18\}$ is more than the distance between the non-hub node $\{22\}$ and the hub node $\{11\}$; however, the node $\{22\}$ is assigned the hub node $\{18\}$. This issue can convert hub nodes

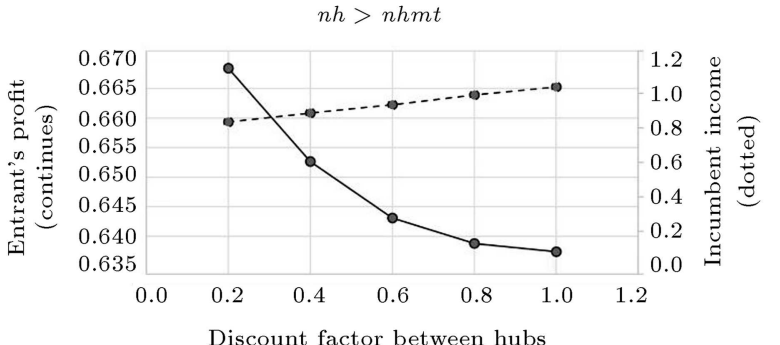

(a)

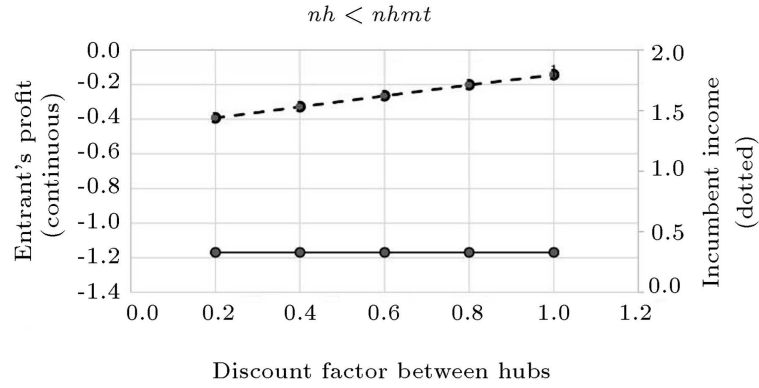

(b)

Figure 8. Entrant's profit and the incumbent's income by changing the discount factor between hubs at $\Theta=7.7$. 


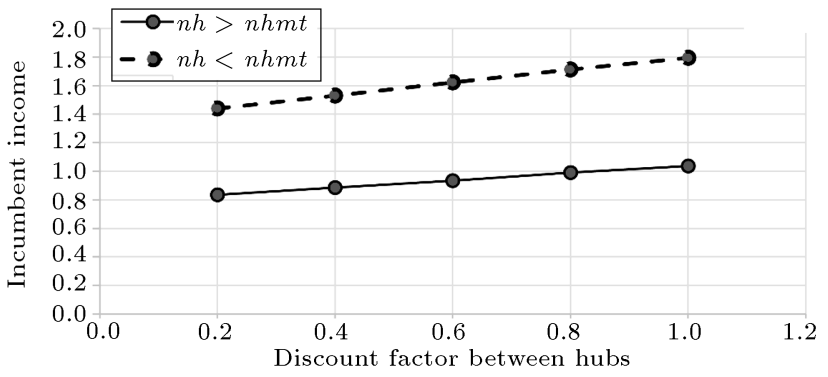

Figure 9. The incumbent income versus discount factor between hubs at $\Theta=7.7$.

into mini-hub and major-hub. It should also be noted that in $(n h>n h m t)$, the entrant's profit is only in $\Theta=\{3.85,5.78\}$ more than the incumbent's income; in other words, incumbent gets a major share of the market by increasing customer's sensitivity to the entrant's prices and accordingly, its income increases. Finally, the environmental costs caused by the speed of the entrant's vehicles in $(n h<n h m t)$ are more than $(n h>n h m t)$, which could be because of distance and vehicle speed. Thus, the entrant requires more hubs than the incumbent to reduce environmental costs and increase the profits.

According to the literature of hub location, there is a discount factor between two hub nodes. In the first mode $(n h>n h m t)$, the total costs, time, and the number of trips are reduced, although the fixed cost of using more hubs is added to the total costs.

Finally, in the two modes $(n h>n h m t)$ and $(n h<n h m t)$, we select entrant's and incumbent's hub nodes based on the map and the best coverage
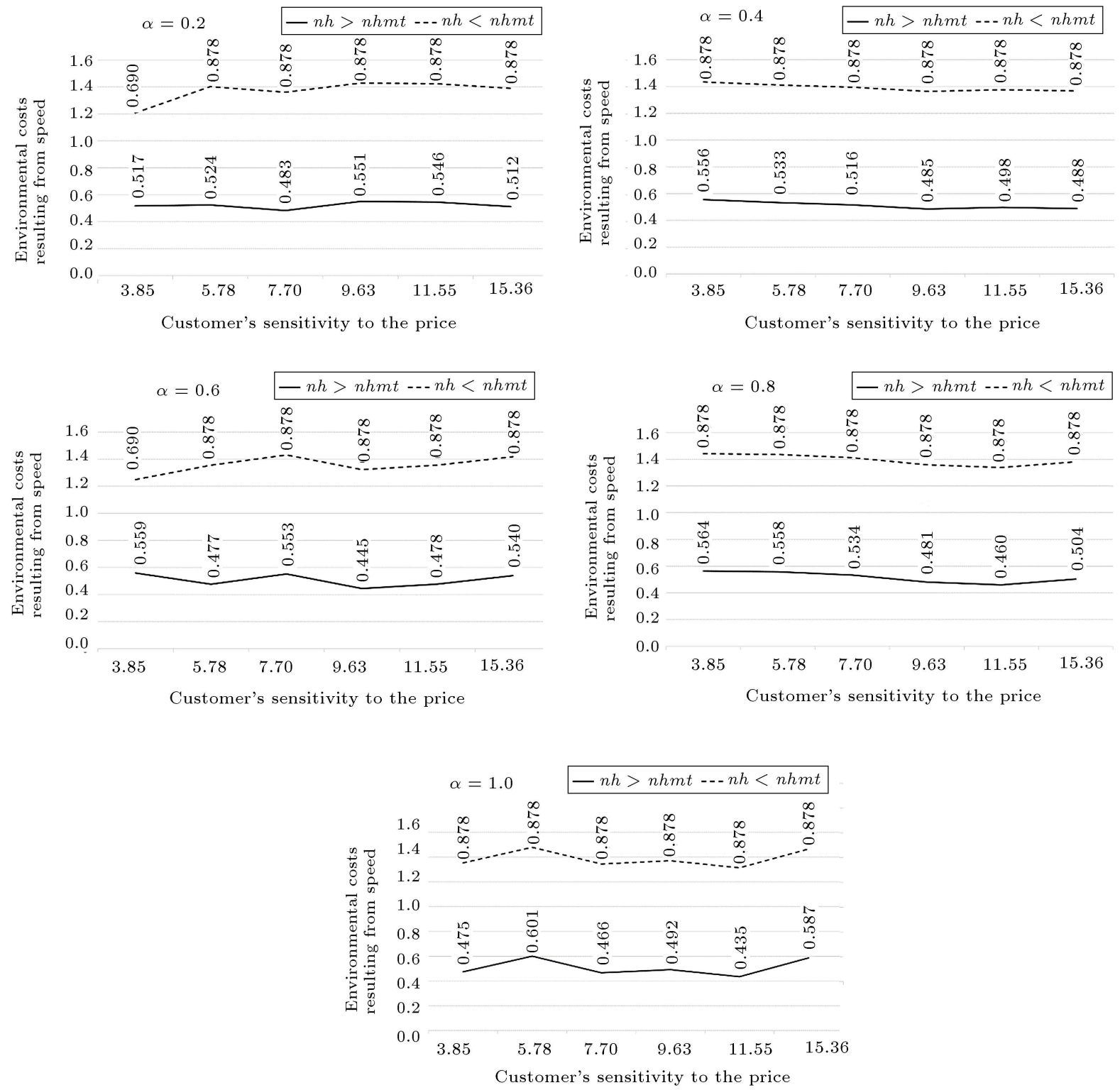

Figure 10. Environmental costs caused by the speed in $(n h>n h m t)$ and $(n h<n h m t)$. 


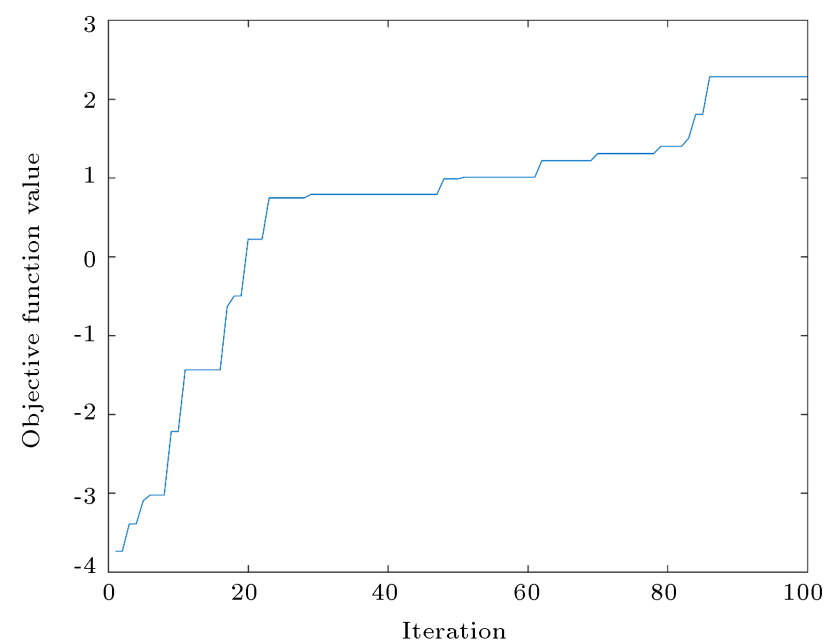

Figure 11. Convergence plot for $(n h>n h m t)$ with 25 nodes: $\alpha=0.2$ and $\Theta=3.85$.

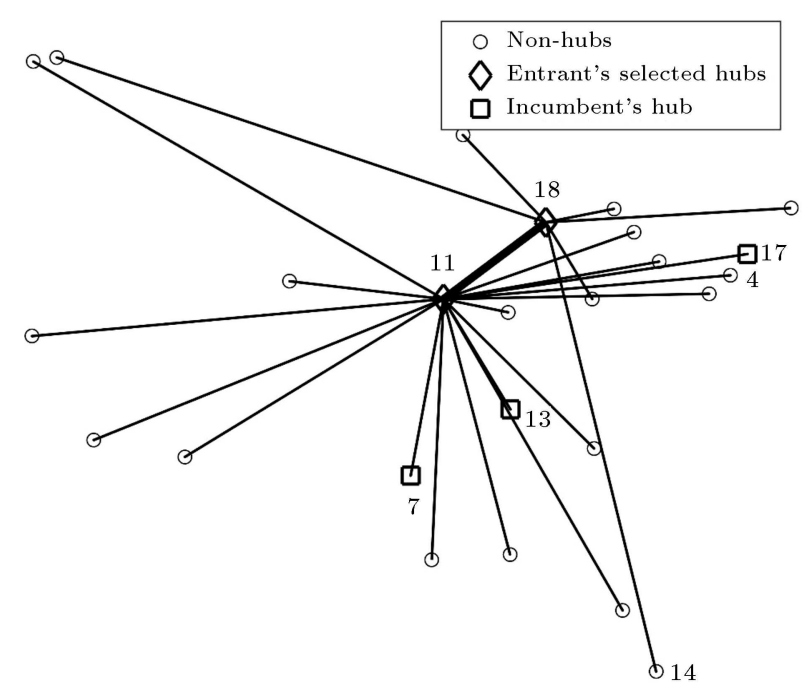

Figure 12. Solution for $(n h>n h m t)$ with 25 nodes: $\alpha=0.2$ and $\Theta=3.85$.

of all nodes. Also, in selecting hub nodes, we consider that a less green space exists in these nodes and the ones around to cause a greater reduction in the environmental pollution.

\section{Conclusion}

This study considered a pricing problem and Hub Location Problem (HLP) together with environmental costs, which is a mixed-integer non-linear programming problem. This problem was solved with Genetic Algorithm (GA) and the results were analyzed from the following perspectives:

1. The impact of $\alpha$ and $\Theta$ on the entrant's profit and the incumbent's income;

2. The ratio of the number of entrant's hubs to the number of incumbent's hubs.
The results show that the larger the number of potential entrant's hubs than the incumbent, the lower the entrant's gain as the discount factor between hub nodes increases. Therefore, managers should consider this important observation on their decision making. Also, if the number of potential entrant's hub is higher than the incumbent, for all discount factors, the customer's profitability decreases upon increasing the price sensitivity. In each price sensitivity coefficient, the incumbent's income increases as the discount factor increases, and the incumbent income is higher when the number of incumbent's hub is more than the potential entrant's hub. Finally, when the number of the potential entrant's hub is more than the incumbent's hub, the environmental costs of the entrant are less than another case; thus, the entrant must use more hubs than the incumbent to reduce environmental costs and increase profits.

Moreover, in this research, customer choice behavior was modeled based on an Logit Model (LM). This behavior depends only on the price of each path. To ensure greater compatibility with the real world, this study can be conducted using a multinomial LM. Furthermore, in this model, customer behavior can be affected by the transportation time or the number of stops (hubs), in addition to the price. Also, an interesting future research direction is to consider a capacity for hubs and hub arc threshold. Also, further studies regarding the role of other metaheuristic algorithms for larger size instances would be worthwhile.

\section{References}

1. Haron, A. "Factors influencing pricing decisions", Int. J. Econ. \& Manag. Sci., 5(1), pp. 312-315 (2016).

2. Gelareh, S., Monemi, R.N., and Nickel, S. "Multiperiod hub location problems in transportation", Transport Res E-Log, 75(1), pp. 67-94 (2015).

3. Karimi, H. and Setak, M. "A bi-objective incomplete hub location-routing problem with flow shipment scheduling", Appl. Math. Model, 57(1), pp. 406-431 (2018).

4. Sarkis, J. and Sundarraj, R.P. "Hub location at digital equipment corporation: a comprehensive analysis of qualitative and quantitative factors", Eur. J. Oper. Res., $137(2)$, pp. 336-347 (2002).

5. McKinnon, A. " $\mathrm{CO}_{2}$ emissions from freight transport in the UK", In Transport and Climate Change, 2, T. Reley, L. Chapman, Emerald Group Publishing Limited, New York (2007).

6. Chen, S. and Shiyi, H. "Pricing for the clean air: Evidence from Chinese housing market", J. Clean Prod., 206(1), pp. 297-306 (2019).

7. Bektas, T. and Laporte, G. "The pollution-routing problem", Transport Res B-Meth, 45(8), pp. 12321250 (2011). 
8. Garrow, L.A., Discrete Choice Modelling and Air Travel Demand: Theory and Applications, 5, UK, Georgia Institute of Technology, USA (2016).

9. Rayfield, W.Z., Rusmevichientong, P., and Topaloglu, H. "Approximation methods for pricing problems under the nested logit model with price bounds", Informs J. Comput., 27(2), pp. 335-357 (2015).

10. Beckmann, M.J. and Thisse, J.F. "The location of production activities", In Handbook of Regional and Urban Economics, Elsevier, 1, pp. 21-95 (1987).

11. Goldman, A. "Optimal location for centers in a network", Transport Sci., 3(4), pp. 352-360 (1969).

12. O'Kelly, M.E. "The location of interacting hub facilities", Transport Sci, 20(2), pp. 92-106 (1986).

13. Campbell, J.F., Ernst, A.T., and Krishnamoorthy, M. "Hub arc location problems: Part I-Introduction and results", Manage Sci., 51(10), pp. 1540-1555 (2005).

14. Campbell, J.F., Ernst, A.T., and Krishnamoorthy, M. "Hub arc location problems: Part II-formulations and optimal algorithms", Manage Sci., 51(10), pp. 15561571 (2005).

15. Campbell, J.F. "Hub location for time-definite transportation", Comput. Oper. Res., 36(12), pp. 31073116 (2009).

16. Nickel, S., Schöbel, A., and Sonneborn, T. "Hub location problems in urban traffic networks", In Mathematics Methods and Optimization in Transportation Systems, P. Niittymäki, J., pp. 95-107, Kluwer Academic Publishers (2001).

17. Gelareh, S. and Nickel, S. "Hub location problems in transportation networks", Transport Res. E-Log, 47(6), pp. 1092-1111 (2011).

18. Adler, N. and Smilowitz, K. "Hub-and-spoke network alliances and mergers: Price-location competition in the airline industry", Transport Res B-Meth, 41(4), pp. 394-409 (2007).

19. Hansen, P., Peeters, D., and Thisse, J. "Facility location under zone pricing", J. Regional Sci., 37(1), pp. 1-22 (1997).

20. Chu, C.Y.C. and Lu, H. "The multi-store location and pricing decisions of a spatial monopoly", Reg. Sci. Urban Econ., 28(3), pp. 255-281 (1998).

21. Zhang, Y. "Designing a retail store network with strategic pricing in a competitive environment", Int. J. Prod. Econ., 159(1), pp. 265-273 (2015).

22. Lüer-Villagra, A. and Marianov, V. "A competitive hub location and pricing problem", Eur. J. Oper. Res., 231(3), pp. 734-744 (2013).

23. Setak, M., Sadeghi-Dastaki, M., and Karimi, H. "Investigating zone pricing in a location-routing problem using a variable neighborhood search algorithm", Int. J. Eng., 28(11), pp. 1624-1633 (2015).

24. Karimi, H. and Setak, M. "Proprietor and customer costs in the incomplete hub location-routing network topology", Appl. Math. Model, 38(3), pp. 1011-1023 (2014).
25. Korani, E., Eydi, A., and Nakhai, I. "Reliable hierarchical multimodal hub location problem: Models and Lagrangian relaxation algorithm", Scientia Iranica, 27(3), pp. 1525-1543 (2020). DOI: 10.24200/sci.2018.50797.1870

26. Daniel, J. and Harback, K.T. "Pricing the major US hub airport", J. Urban Econ., 66(1), pp. 33-56 (2009).

27. Spiller, P.T. "A note on pricing of hub and spoke networks", Econ. Lett., 30(2), pp. 165-169 (1989).

28. Daniel, J.I. "Congestion pricing and capacity of large hub airports: A bottleneck model with stochastic queues", Econometrica, 63(2), pp. 327-370 (1995).

29. Oum, T., Zhang, A., and Zhang, Y. "A note on optimal airport pricing in a hub-and-spoke system", Transport Res B-Meth, 30(1), pp. 11-18 (1996).

30. Nero, G. and Black, J. "Hub-and-spoke networks and the inclusion of environmental costs on airport pricing", Transport Res D-Trans Env., 3(5), pp. 275296 (1998).

31. Vowles, T.M. "Airfare pricing determinants in hubto-hub markets", J. Transp. Geogr., 14(1), pp. 15-22 (2006).

32. O'Kelly, M.E. "Fuel burn and environmental implications of airline hub networks", Transport Res D-Trans Env., 17(7), pp. 555-567 (2012).

33. Loo, B.P.Y., Linna, L., Voula, P., et al. " $\mathrm{CO}_{2}$ emissions associated with hubbing activities in air transport: an international comparison", J. Transp Geogr., 34, pp. 185-193 (2014).

34. Niakan, F., Vahdani, B., and Mohammadi, M. "A multi-objective optimization model for hub network design under uncertainty: An inexact rough-interval fuzzy approach", Eng. Opt., 47(12), pp. 1670-1688 (2015).

35. Zhalechian, M., Tavakkoli-Moghaddam, R., Rahimi, Y., et al. "An interactive possibilistic programming approach for a multi-objective hub location problem: Economic and environmental design", Appl. Soft. Comput., 52, pp. 699-713 (2017).

36. Zhang, S., Derudder, B., and Witlox, F. "The impact of hub hierarchy and market competition on airfare pricing in US hub-to-hub markets", J. Air Transp. Manag., 32(2), pp. 65-70 (2013).

37. Lin, M. and Zhang, A. "Hub congestion pricing: Discriminatory passenger charges", Econ of Transp, 5(1), pp. 37-48 (2016).

38. Lin, M.H. and Zhang, Y. "Hub-airport congestion pricing and capacity investment", Transport Res BMeth, 101(1), pp. 89-106 (2017).

39. Cunha C.B. and Silva, M.R. "A genetic algorithm for the problem of configuring a hub-and-spoke network for a LTL trucking company in Brazil", Eur. J. Oper. Res., 179(3), pp. 747-758 (2007).

40. Topcuoglu, H., Corut, F., Ermis., M., et al. "Solving the uncapacitated hub location problem using genetic algorithms", Comput. Oper. Res., 32(4), pp. 967-984 (2005). 
41. Rudolph, G. "Convergence analysis of canonical genetic algorithms", IEEE Trans. Neural Networks, 5(1), pp. 96-101 (1994).

42. O'kelly, M.E. "A quadratic integer program for the location of interacting hub facilities", Eur. J. Oper. Res., 32(3), pp. 393-404 (1987).

43. Calik, H., Alumur, S., Kara, B., et al. "A tabusearch based heuristic for the hub covering problem over incomplete hub networks", Comput. Oper. Res., 36(12), pp. 3088-3096 (2010).

44. Akçelik, R. and Besley, M. "Operating cost, fuel consumption, and emission models in aaSIDRA and aaMOTION", 25th Conference of Australian Institute of Transport Research (2013).

45. Genta, G., Motor Vehicle Dynamics: Modeling and Simulation, World Scientific, Italy (1997).

\section{Biographies}

Mohaddese Mohammadi received her BSc and MSc degrees in Industrial Engineering from Babol Noshirvani University of Technology in 2014 and University of Bojnord in 2017, respectively. Her research interests are facility location, optimizations, mathematical modeling, and integer programming.

Hossein Karimi is the Head of Industrial Engineering Department at the University of Bojnord. He completed his BSc, MSc, and $\mathrm{PhD}$ degrees at the University of Bojnord, Shahed University, and K.N.T. University of Technology, respectively. He is the author of several journal papers on hub location problem, vehicle routing problem, and multiple attribute decision-making. In addition, he wrote three academic books in Persian about location problems and integer programming. 